The difficulty lies in implementing what we know. How can we splice safety culture and practice into the genome of health care?

Improving safety of patients should be one of the highest priorities of healthcare leaders. Perhaps things are changing. In the UK the National Patient Safety Agency has just been set up, and in the USA President Bush has increased the budget of the Agency for Healthcare Research and Quality by $\$ 150 \mathrm{~m}$ to promote research on safety of patients. ${ }^{12}$

Easy access to research on improving safety may help health professionals and managers to make care safer. This journal has included papers on the safety of health care and on clinical risk management in the past. But, to reflect the increasing concern about the endemic "unsafeness" of healthcare systems worldwide and the need to find ways to reduce risk and, with that, the incidence of adverse events and harm suffered by patients, the journal will expand and, from March 2002, become Quality and Safety in Health Care. The new journal will also look different. The logo will change; the cover will be grey and green with the contents listed on the inside on the first page; and the layout will be different. We hope that these changes to the format of the journal will make it easier for readers. We will continue to publish as many papers on quality improvement, but each issue will include more papers on safe care and safe practice. We invite readers to send us these. Changing attitudes and practices will be hard work. Patients are being placed at unnecessary risk and many are harmed; they expect that we will offer safer care. We believe that safety and quality will be the Holy Grail of medicine in the 21 st century.

PAUL BARACH

Editor, Quality in Health Care

Center for Patient Safety, Department of Anesthesia and Critical Care,

University of Chicago, Chicago, IL 60637, USA

pbarach@airway.uchicago.edu

FIONA MOSS

Editor in Chief, Quality in Health Care

fmoss@londondeanery.ac.uk

1 Brennan TA, Leape LL, Laird L, et al. Incidence of adverse events and negligence in hospitalized patients: results of the Harvard Medical Practice Study I. N Engl f Med 1991;324:370-6.

2 Wilson RM, Runciman WB, Gibberd RW, et al. The quality in Australian healthcare study. Med f Aust 1995;163:458-71.

3 Vincent C, Neale G, Woloshynowych M. Adverse events in British hospitals: preliminary retrospective record review. BMF 2001;322:501-2.

4 Institute of Medicine. To err is human: building a safety health system. Washington, DC: National Academy Press, 1999.

5 Department of Health. An organisation with a memory. Report of an expert group on learning from adverse events in the NHS. www.doh.gov.uk/ orgmemreport/ (accessed 7 September 2001).

6 Committee on Quality of Health Care in America, Institute of Medicine. Crossing the quality chasm: a new health system for the 21st century. Washington, DC: National Academy Press, 2001.

7 Department of Health. Building a safer NHS for patients. Implementing an organisation with a memory. www.doh.gov.uk/buildsafenhs/ (accessed 7 September 2001)

8 Bates DW, Cullen DJ, Laird N, et al. Incidence of adverse drug events and potential adverse drug events: implications for prevention. ADE Prevention Study Group. $7 A M A$ 1995;274:29-34.

9 Reason J. Managing the risks of organizational accidents. Aldershot, Reason J. Managing the

Waik KE. Organizatiol culture as a source of high reliability. Calif Manage Rev 1987;24:112-27.

11 Donaldson MS, Mohr JJ. Exploring innovation and quality improvement in health care micro-systems: a cross case analysis. Washington, DC: National Academy Press, 2000

12 Report of the Quality Interagency Coordination Task Force (QuIC) to the President. Doing what counts for patient safety: federal actions to reduce medical errors and their impact. www.Quic.org

\title{
Challenges to quality monitoring systems in care homes
}

In the UK access to continuing care services is often a gamble and, as consumers of health care, older people have had little choice in where and how these services are provided as the continuing care of many older persons has shifted from the health service to the independent sector. This shift has raised concerns about the quality of care in private nursing homes and has placed the need to determine such quality high on the government's policy agenda. The traditional quality assurance mechanism has relied on a registration and inspection system whereby local government authorities monitor and review service delivery. However, the processes are often bureaucratic and ineffective. More recently, the Care Standards Act (2000) and the Regulation of Care (Scotland) Act 2001 have set out the new regulatory framework for all care homes in the UK. This will provide national standards of registration and inspection, with increased authority for health and social care agencies to take action when poor quality is suspected or detected.

A registration and inspection system can only ever provide one component of a quality system for the continuing care of older people. Research by Wagner et $a l^{1}$ reported in this issue of Quality in Health Care highlights the difficulties in determining the most appropriate mechanism for monitoring quality in care homes, particularly when the emphasis is on "care outcomes". The authors point out that it is difficult to determine the impact of quality systems on the quality of care of residents and conclude that there needs to be a greater emphasis on the qualitative aspects of care and, in particular, improvement in the measurement of quality of life.
Outcome can be defined as the end result of care, but outcomes do not directly assess quality of performance; they only permit inferences to be made about the quality of the processes of care. The focus on outcomes is not always appropriate in the context of continuing care. In much of healthcare provision a focus on "health gain"- that is, that the intervention results in a gain in health status for the patient-is seen as the most important outcome measure. In this issue of Quality in Health Care Meiland et al discuss the complexity of measuring outcome in residents of care homes. By assessing the burden on caregivers, the problems of delayed admission to care homes were identified as well as the continuing effect of these problems on the caregiver following admission of the patient. This suggests that measuring outcome with the patient alone only provides one part of the total picture. Outcomes in continuing care for older people should focus on increasing quality of life rather than longevity. Because quality of life is difficult to define and even more difficult to measure, particularly with physically and mentally frail people, outcomes from care inputs cannot always be clearly articulated as highlighted by Meiland et al. Furthermore, the care of older people in continuing care settings is predominantly based on the maintenance of a normal pattern of lifestyle. However, as pointed out by Wagner et al, such individualised patterns of lifestyle are difficult to measure using standardised instruments. This is not to argue that quality measurement in care homes should not focus on hard outcome data, but that it also needs to include subjective interpretation of quality of life and individual desire and 
ability. The emphasis should be on the individual's potential to achieve his or her desired realistic health choices.

However, the most interesting finding of the research by Wagner et al is that, despite the dominant rhetoric of user involvement, "the opinion of residents was seldom used to evaluate the effectiveness of quality systems". Who better to decide on the effectiveness of the end result of care inputs than the patients and others significant to them? In an era where individuals are encouraged to be experts in their own health and care needs, surely we should focus our energies on finding ways to enable older people and their supporters to identify changes in their health status and to establish self-report mechanisms for them to report satisfaction with the care and its outcomes. Reed et $a l^{3}$ reported on one such system ("Qual A Sess") whereby care homes engaged in a self-assessment of quality and included care home residents as self-assessors. If approaches such as this are to become the norm, then substantial investment is needed to enable older people to have such a voice. If we are going to be committed to enabling older people to plan their own destiny, we need to see investment in local models of peer review and quality improvement in addition to central government models of quality control.

However, there remains a paucity of high quality research with older people in care homes ${ }^{4}$ and, as such, there is little understanding of the most effective ways to deliver such services and measure their impact. If the new national framework of regulation is to be effective, a concerted effort must be made to understand the dynamics of care homes and the desired care outcomes of residents, as well as high levels of investment in staff development and continuing professional development. National standards can only ever be a blunt instrument to measure quality and, if the services for older people in care homes are to be consistently of a high standard, then the empowerment of care home staff and residents to determine their own quality destiny has to be part of the process.

B MCCORMACK

Professor of Nursing Research,

University of Ulster/Director of Nursing Research and Practice

Development,

Royal Hospitals Trust,

Belfast, UK

brendan.mccormack@royalhospitals.n-i.nhs.uk

H MCKENNA

Professor and Head of School of Nursing

School of Health Sciences,

University of Ulster,

fordanstown, UK

hp.mckenna@ulst.ac.uk

1 Wagner C, van der Wal G, Groenewegen PP, et al. The effectiveness of quality systems in nursing homes: a review. Quality in Health Care 2001;10:211-7.

2 Meiland FJM, Danse JAC, Wendte JF, et al. Burden of delayed admission to psychogeriatric nursing homes on patients and their informal caregivers. Quality in Health Care 2001;10:218-23.

3 Reed J, Cook G, Stanley D. Promoting partnership with older people through quality assurance systems: issues arising in care homes. NT Res 1999;4:353-63.

4 Royal College of Physicians, Royal College of Nursing, and British Geriatric Society. The health and care of older people in care homes: an interdisciplinary approach. London: Royal College of Physicians, 2000.

\section{Improving teaching about improving practice}

There is increasing evidence that the structured use of the principles and methods of continuous quality improvement (CQI) in healthcare settings will have a positive effect on improving care. ${ }^{1-3}$ Recognition of this in the UK can be seen from frequent references to CQI in Government publications driving the "modernisation agenda". Government support of centrally funded initiatives such as the National Breakthrough Collaboratives ${ }^{4}$ provides additional evidence, although it is not clear whether a common understanding exists of what are its key elements. For example, these should include a focus on improving the way we understand and meet the needs of patients/users; a focus on improving the processes by which their care is delivered; and the application of improvement methodology that enables us to learn as we go. Finally, it is essential that the delivery of care is improved by the interprofessional teams who provide it.

It is still early days and the limited availability of knowledge and expertise in these methods means that improvement projects often have to rely on the use of external facilitators for their successful conclusion. It has been suggested that it is unlikely that success will be achieved without such facilitation, ${ }^{5}$ although it is also true to say that such dependency may itself sow the seeds of future failure. Resourcing such facilitation in the long term is not sustainable if we really want to see improvement become a routine part of everyday practice.

We therefore have to consider how to develop the necessary knowledge and skills within health care itself and, in particular, how to help practitioners learn improvement skills alongside their professional and technical skills. ${ }^{6}$ In this issue of Quality in Health Care Kyrkjebø et al describe an educational project that addresses this crucial question and, in doing so, they make an important contribution to the work of others in the field. ${ }^{8-10}$

This work is beginning to integrate understanding about best improvement practice with knowledge of best educational practice. Making them both practice based and relevant to patients and students produces the best improvements and the best learning. Such an aim underlies the project reported by Kyrkjebø and colleagues. ${ }^{7}$ They tested out a student experience that was carefully designed to have a patient focus, to link theory and learning in practice, and to introduce students to improvement methods that could make a real difference to the quality of care. Although they were unable to provide a formal interprofessional team experience, the students were able to develop insight into skills needed to work jointly with colleagues.

As the study infers, such learning must go beyond simply learning the mechanics of improvement tools. Stories of successful improvement are inevitably stories of people learning together and, in health care, this means within clinical and other practice based settings. We need to establish creative learning strategies that involve students in real work as a learning medium so that they may leave something behind as well as take something away. ${ }^{11}$ They need to develop a personal understanding that, to be continuous improvers, they must be continuous learners. In educational terms this reflects the difference between "deep" and "surface" learning, ${ }^{12}$ and reinforces the need for deep learning that develops each student's self-concept 